\title{
The effects of microchipping C57BL/6N mice on standard
}

\section{phenotyping tests [version 1; peer review: 1 approved, 1}

\section{approved with reservations]}

\author{
R.S. Bains (D) H.L. Cater, M. Stewart, C.L. Scudamore, S.E. Wells \\ MRC Harwell Institute, Mary Lyon Centre, Harwell Campus, Oxfordshire, OX11 ORD, UK
}

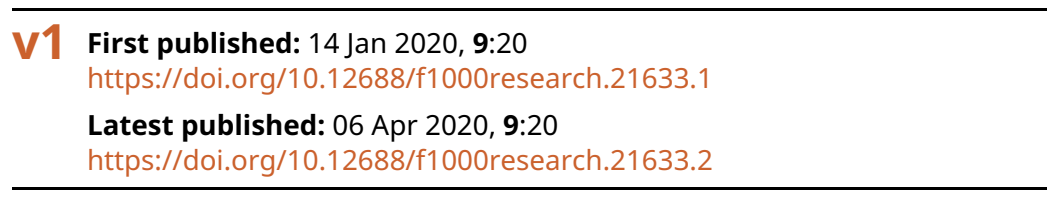

\section{Abstract}

The C57BL/6N inbred lines of mice are widely used in genetic research. They are particularly favoured in large scale studies such as the International Mouse Phenotyping Consortium (IMPC), where C57BL/6N mice are genetically altered to generate a collection of null alleles (currently more than 8500 null alleles have been generated). In this project, mice carrying null alleles are subjected to a pipeline of broad-based phenotyping tests to produce wide ranging phenotyping data on each model. We have previously described the development of a Home Cage Analysis system that automatically tracks the activity of group housed mice from a microchip inserted in the groin. This platform allows assessment of multiple biologically relevant phenotypes over long periods of time without experimenter interference, and therefore is particularly suited for high through-put studies. To investigate the impact of microchips on other tests carried out in the IMPC pipeline, we inserted microchips in 12 male and 12 female C57BL/6Ntac mice at seven weeks of age. Starting at nine weeks of age these mice underwent standard phenotyping tests, concurrently with 20 unchipped C57BL/6Ntac mice (10 females, 10 males). Tissues from a subset of the microchipped mice (six males and six females), chosen at random, were also sent for histopathological examination at the end of the phenotyping pipeline.

\section{Keywords}

RFID, Microchips, phenotyping, C57BL/6NTac, mice, Home cage analysis, histopathology

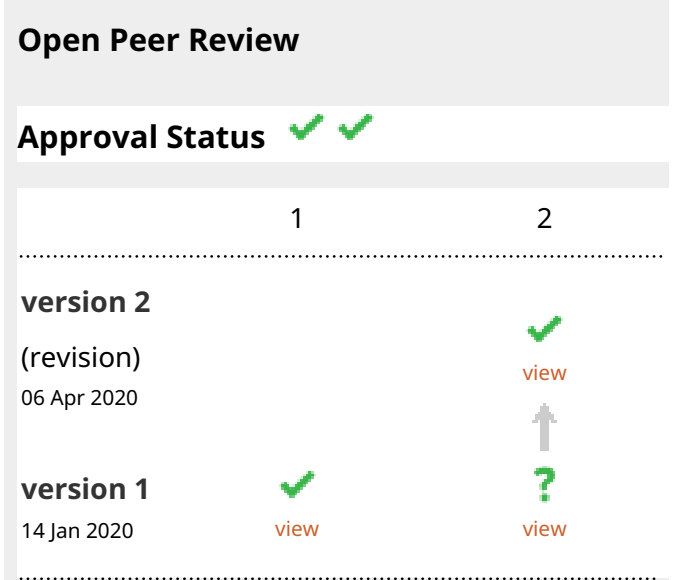

1. Jonathan M. Williams, Royal Veterinary

College, North Mymms, UK

2. Helmut Fuchs iD, Helmholtz Zentrum München, Neuherberg, Germany

Annemarie Zimprich (D), Institute of

Developmental Genetics, Helmholtz Zentrum

München, Neuherberg, Germany

Gregor Miller, Institute of Experimental

Genetics, Helmholtz Zentrum München,

Neuherberg, Germany

Manuela Östereicher, Institute of

Experimental Genetics, Helmholtz Zentrum

München, Neuherberg, Germany

Any reports and responses or comments on the article can be found at the end of the article. 
Corresponding author: R.S. Bains (r.bains@har.mrc.ac.uk)

Author roles: Bains RS: Data Curation, Formal Analysis, Investigation, Methodology, Project Administration, Visualization, Writing Original Draft Preparation; Cater HL: Conceptualization, Investigation, Methodology, Project Administration, Supervision, Writing Review \& Editing; Stewart M: Data Curation, Formal Analysis, Methodology, Validation, Writing - Review \& Editing; Scudamore CL: Formal Analysis, Investigation, Writing - Review \& Editing; Wells SE: Conceptualization, Funding Acquisition, Resources, Supervision, Writing - Review \& Editing

Competing interests: No competing interests were disclosed.

Grant information: This work was supported by the Medical Research Council [A410-Mary Lyon Centre].

The funders had no role in study design, data collection and analysis, decision to publish, or preparation of the manuscript.

Copyright: (c) 2020 Bains RS et al. This is an open access article distributed under the terms of the Creative Commons Attribution License, which permits unrestricted use, distribution, and reproduction in any medium, provided the original work is properly cited.

How to cite this article: Bains RS, Cater $\mathrm{HL}$, Stewart $\mathrm{M}$ et al. The effects of microchipping C57BL/6N mice on standard phenotyping tests [version 1; peer review: 1 approved, 1 approved with reservations] F1000Research 2020, 9:20

https://doi.org/10.12688/f1000research.21633.1

First published: 14 Jan 2020, 9:20 https://doi.org/10.12688/f1000research.21633.1 


\section{Introduction}

The overarching aim of phenotyping studies is to understand and describe the relationship between genotype and phenotype. However, mouse behaviour is variable, dynamic and adaptive and therefore is influenced by a variety of genetic and environmental factors such as motivation, interaction with the experimenter, experimental design, test order, testing time and environmental enrichment $^{1,2}$. In recent years, automated home cage phenotyping as a complement to conventional out-of-cage testing has increasingly been used to enrich phenotype datasets ${ }^{3}$. We have been developing a novel Home Cage Analysis system (HCA) for multiply housed mice that is entirely compatible with the modern high density individually ventilated caging (IVC) systems. The HCA allows us to record automated detailed behavioural parameters over time in an undisturbed standard rack-mounted cage - the type the mice are born, reared and constantly housed in - within their established social groups. The HCA combines radio frequency identifier (RFID) tracking with video recording for automated behavioural scoring. For optimal resolution of the RFID signals from the microchips, the chips are inserted subcutaneously in the animal's groin ${ }^{4}$. Though designed to investigate subtle phenotypes in undisturbed animals, thereby reducing variability, it is possible that the insertion of the RFID microchip may itself influence behaviour, thus changing the phenotyping profile.

Implantable micro-identification has been used as an effective means of identification in long term rodent studies for over two decades; where a hermetically sealed inert glass cylinder, $12 \mathrm{~mm}$ in length and $2 \mathrm{~mm}$ in diameter, containing the microchip transponder is implanted subcutaneously in the mid dorsal region of the animal ${ }^{5,6}$. Although widely considered safe, some long term studies have reported the development of foreign-body-induced tumours in various mouse strains ${ }^{7,8}$. Furthermore, the subcutaneous implantation of the microchip in the groin requires the mice to be anaesthetised; although gaseous anaesthesia is brief (less than one minute), this may interfere with the phenotyping tests further downstream ${ }^{9}$. The implant itself may also confound certain assays such as dual-energy X-ray absorptiometry (DEXA) which measures total body composition. The current study investigates phenotypic effects of subcutaneous microchip implants in the groin of C57BL/6NTac mice. At the end of the study the tissue around the microchip was sent for histopathology to detect any signs of foreign-body induced pathology.

\section{Methods}

\section{Ethics statement}

All procedures and animal studies were carried out in accordance with the Animals (Scientific Procedures) Act 1986, UK, Amendment Regulations 2012 (SI 4 2012/3039). This study has been approved by the Animal Welfare and Ethical Review Board (AWERB), resulting in licence 30/2890. All efforts were made to minimize suffering by considerate housing and husbandry, the details of which are available at the IMPC portal: http://www.mousephenotype.org/about-impc/arrive-guidelines. Animal welfare was assessed routinely for all mice involved.

Adult mice were killed by terminal anaesthesia followed by exsanguination, and death was confirmed by either cervical dislocation or cessation of circulation.

\section{Husbandry}

22 C57BL/6NTac male and 22 C57BL/6NTac female mice, bred at the Mary Lyon Centre, Harwell, were housed in IVC's, on Eco-pure spruce chips grade 6 bedding (Datesand, U.K.), with shredded paper shaving nesting material and small cardboard play tunnels for enrichment. Mice were randomly assigned to groups of three to five mice per cage and the phenotyping tests were carried out as a part of a bigger batch of experimental animals and their position in the batch was randomly allocated. While the method of randomisation was simple, the experimenters were blinded to the test groups. Only the experimenters carrying out X-ray analysis after the test would know the groups as that would not be possible to blind. The mice were kept under controlled light (light 7 a.m. to 7 p.m., dark 7 p.m. to 7 a.m.), temperature $\left(21{ }^{\circ} \mathrm{C} \pm 2{ }^{\circ} \mathrm{C}\right)$ and humidity $(55 \% \pm 10 \%)$ conditions. They had free access to water (25 p.p.m. chlorine) and were fed ad libitum on a commercial diet (SDS Rat and Mouse No.3 Breeding diet (RM3)).

Animal welfare checks were carried out visually once a day.

\section{Microchipping}

The microchipping procedure has been described in Bains et al., $2016^{4}$. Briefly, RFID microchips were injected subcutaneously into the lower left or right quadrant of the abdomen of each mouse at seven weeks of age. These microchips were contained in standard ISO biocompatible glass capsules $(11.5 \times 2 \mathrm{~mm}$, PeddyMark Ltd. UK). The procedure was performed on sedated mice (Isoflo, Abbott, UK) after topical application of local anaesthetic cream on the injection site prior to the procedure (EMLA Cream 5\%, AstraZeneca, UK). While using local anaesthetic is not common practice, we have found that a combination of these anaesthetics is the most refined method of delivering these scientific data, as this minimizes any acute discomfort the animal may feel after recovering from the general anaesthetic.

No sutures were required.

\section{Phenotyping pipeline}

12 male and 12 female C57BL/6NTac mice were microchipped in the groin at seven weeks of age and phenotyped alongside 10 male and 10 female C57BL/6NTac baseline mice $(n=24-20)$, starting at nine weeks of age. 20 baseline and 24 microchipped mice underwent systematic broad-based phenotyping for adults, according to the pipeline described in Table 1. The reason for the difference in numbers in each experimental group was due to the HCA being optimised for three animals in a home cage while the mice in the standard phenotyping pipeline are housed in groups of five. A sample size of 10 mice per sex was calculated based on an alpha of $1 \%$ and power of 0.8 . All standard operating procedures have been described within the International Mouse Phenotyping Resource of Standardised Screens, the IMPReSS database (www.mousephenotype.org/impress).

To provide traceability, IMPReSS also stores a record of change histories, all tests in this study were carried out at the Mary Lyon Centre, MRC Harwell Institute, between November 2015 and March 2016. 


\begin{tabular}{|c|c|c|}
\hline Table 1. Phenotyping pipeline & & \\
\hline Test & Phenotypic area & (weeks) \\
\hline Body weight & General health and welfare/essential for certain tests & Weekly from 4 to 16 weeks \\
\hline Open Field & Anxiety and exploratory behaviours & 9 \\
\hline Combined SHIRPA and Dysmorphology (CSD) & Neurological/gross morphology & 9 \\
\hline Grip Strength & Musculoskeletal/neurological & 9 \\
\hline Acoustic Startle and Pre-pulse Inhibition & Sensorimotor gating & 10 \\
\hline IPGTT & Metabolic & 13 \\
\hline X-ray & Musculoskeletal & 14 \\
\hline Buditory Brain Stem Response + Click Stimulus & Musculoskeletal and body composition & 14 \\
\hline Slit Lamp/Opthalmoscope & Hearing & 15 \\
\hline Terminal & Vision/eye morphology & 16 \\
\hline
\end{tabular}

The table outlines the phenotyping tests in the pipeline in the order that they are carried out in, along with the age in weeks that each test is conducted. SHIRPA, SmithKline Beecham, Harwell, Imperial College and Royal London Hospital phenotype assessment; IPGTT, intraperitoneal glucose tolerance test; DEXA, dual energy X-ray analysis.

\section{Necropsy and pathology}

At the end of the phenotyping pipeline, six randomly chosen microchipped males and six randomly chosen microchipped females at 16 weeks of age underwent necropsy. The animals were simultaneously randomised without considering any variable as a part of a larger batch of animals undergoing the same procedure. The choice of euthanasia, overdose of anaesthetic followed by exsanguination, was considered the most refined and appropriate for gathering the data for clinical chemistry and haematology assays by the AWERB. The site of microchip implantation was examined and the tissue surrounding the implant was fixed in 10\% neutral buffered formalin (NBF). Tissue samples from the implant sites were processed routinely to wax and $3 \mu \mathrm{m}$ sections were stained with haematoxylin and eosin (H\&E) and examined by a veterinary pathologist.

\section{Data analysis}

Data for Open Field test, Grip Strength test, Acoustic Startle and Pre-pulse Inhibition and DEXA were analysed using the Student's t-test with Welch's correction for unequal variance. The data for weekly weight curve and intraperitoneal glucose tolerance test (IPGTT) were assessed using two-way analysis of variance (ANOVA) with Sidak post hoc analysis. As the current study tests multiple hypotheses, Bonferroni's correction was applied to avoid type 1 error in the interpretation of the data. Therefore, an alpha value of $<0.005$ was calculated for an effect to have statistical significance. The categorical data such as Combined SHIRPA and Dysmorphology (CSD), opthalmology and X-ray were analysed using Fisher's exact test. GraphPad Prism 8.3.0 was used for statistical analysis.

\section{Results}

Phenotyping

Dysmorphology. No evidence of any major differences in morphological features was found between the chipped and unchipped mice. The X-ray analysis showed that microchips were situated at the site of implantation.

Metabolism. There was no significant difference between the weight curve data of chipped mice compared to unchipped mice over the 12 -week testing period ${ }^{10}$. Figure $1 \mathrm{C}$ represents the mean body weight \pm sem of each group compared every week starting at four weeks of age $(16.71 \pm 0.29$ non-chipped vs $16.13 \pm 0.46$ chipped females; $16.5 \pm 0.63$ non-chipped vs $18.22 \pm 0.29$ chipped males), until the end of the study at 16 weeks $(24.22 \pm 0.27$ non-chipped vs $25.1 \pm 0.5$ chipped for females; $31.37 \pm 0.19$ nonchipped vs $30.7 \pm 0.41$ chipped males). DEXA body composition measurements, represented in Figure 1A, showed significant differences between the two groups, when analyzing bone mineral density $(p<0.0001 ;$ mean \pm sem $=0.054 \pm 0.001$ non-chipped vs $0.063 \pm 0.001$ chipped females; $0.057 \pm 0.001$ non-chipped vs $0.067 \pm 0.001$ chipped males) and bone mineral content $(\mathrm{p}<0.001$; $0.49 \pm 0.01$ non-chipped vs $0.56 \pm 0.01$ chipped females; $0.55 \pm 0.01$ non-chipped vs $0.63 \pm 0.01$ chipped males) in both males and females. This can be attributed to the texture of the microchip, which would behave similarly to bone when imaged using DEXA. 
A)

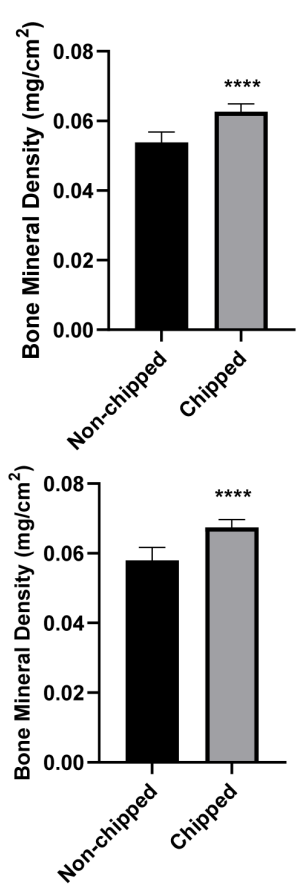

B)

Females

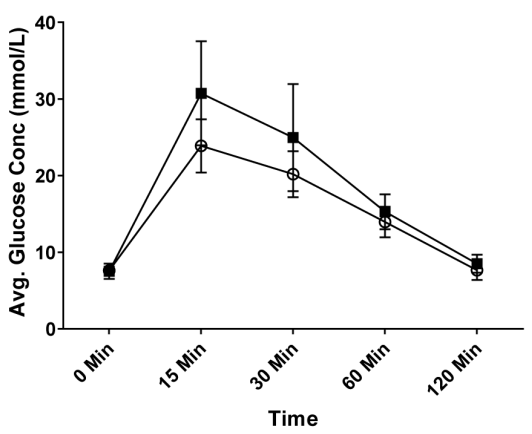

C) Females

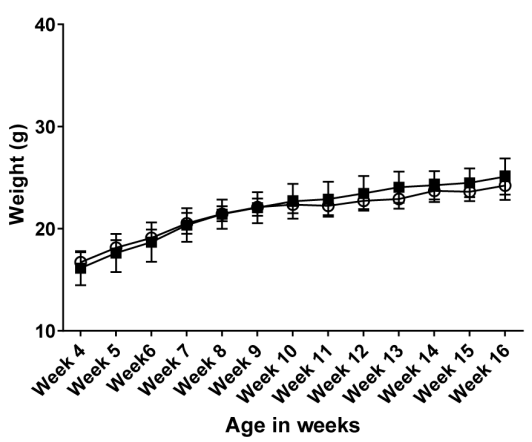

Females

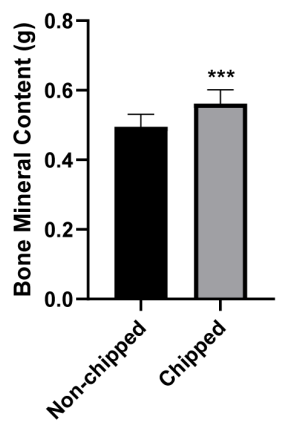

- Non-chipped Chipped

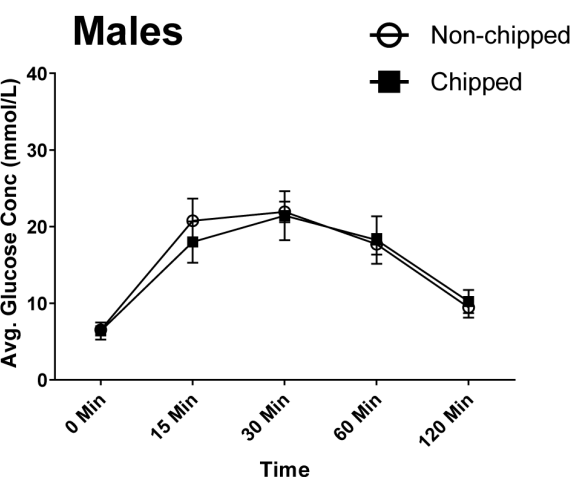

Males

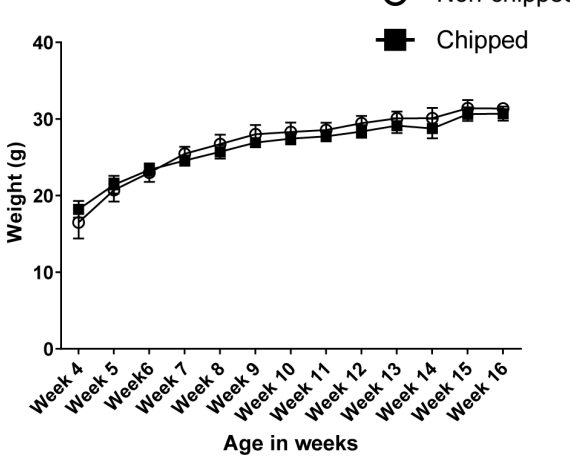

Figure 1. A) Bars represent average bone mineral density $\left(\mathrm{g} / \mathrm{cm}^{2}\right)$ and average bone mineral content $(\mathrm{g})$, for males and females as measured through dual-energy X-ray absorptiometry (DEXA). Microchipped females as well as males show a significant increase in the two parameters as compared to unchipped animals $\left(n=10-12 ;{ }^{* *} \mathrm{p}<0.001\right.$ and $\left.{ }^{* * *} \mathrm{p}<0.0001\right)$, the $T$ bars represent \pm sem. B) The lines represent average glucose concentration $(\mathrm{mmol} / \mathrm{L})$ over time as measured through IPGTT, the shapes represent average glucose concentration $(\mathrm{mmol} / \mathrm{L})$ for each group at that time point, the T bars represent \pm sem. No statistically significant difference was seen in either sex for microchipped versus unchipped mice. C) The lines represent the body weight curve of the two treatment groups over time, the shapes represent mean body weight $(\mathrm{gm})$ for each group at that time point, the T bars represent \pm sem. No statistically significant difference was seen in either sex for microchipped versus unchipped mice. 
The results from the IPGTT, represented in Figure 1B, showed an increase in mean blood glucose concentration at the 15-minute time point for microchipped females $(30.73 \pm 1.87)$ versus unchipped females $(23.88 \pm 1.04)$, but this was not statistically significant. No evidence of altered glucose metabolism was seen in chipped males $(17.99 \pm 0.87)$ versus unchipped males $(20.76 \pm 0.86)$ for the 15-minute time point.

Neurological, behavioral and sensory. No significant differences were observed in the whole arena open field locomotor parameters between chipped and unchipped mice (means \pm sem $=$ $12.08 \pm 0.40$ for non-chipped vs $12.32 \pm 0.36$ chipped females; means \pm sem $=12.06 \pm 0.51$ for non-chipped vs $10.73 \pm 0.19$ chipped for males); this was also true for the locomotor activity observed in the arena during the modified SHIRPA testing, where all animals tested showed normal activity. Grip strength measurements of fore and hind limbs normalized to body weights between the two groups were also non-significant (means \pm sem $=4.89 \pm 0.19$ for non-chipped vs $5.12 \pm 0.19$ chipped females; means \pm sem $=4.91 \pm 0.21$ for non-chipped vs $4.83 \pm 0.24$ chipped for males).

Acoustic startle (means \pm sem of startle amplitude $=481.75 \pm 29.86$ non-chipped vs $443.79 \pm 31.19$ for females; $668.33 \pm 53.71$ nonchipped vs $499.27 \pm 50.39$ chipped males) and startle response to pre-pulse showed no significant difference between the chipped and unchipped mice (means \pm sem of pre pulse1 startle amplitude $=530.64 \pm 48.46$ non-chipped vs $418.6 \pm 23.9$ for females; $659.28 \pm 57.03$ non-chipped vs $504.65 \pm 42.24$ chipped males; means \pm sem of pre pulse2 startle amplitude $=298.03 \pm 19.76$ non-chipped vs $254.7 \pm 18.96$ for females; $315.35 \pm 32.48$ nonchipped vs $275.58 \pm 32.29$ chipped males; means \pm sem of pre pulse 3 startle amplitude $=302.53 \pm 18.37$ non-chipped vs $226.67 \pm 12.7$ for females; $308.27 \pm 33.65$ non-chipped vs $272.91 \pm 32.29$ chipped males.

\section{Pathology}

During the lifespan of the mice, the general health of the mice was considered normal and no clinical symptoms associated with the implant sites were observed.

On microscopic examination of the implant sites, a thin fibrous capsule was identified in the subcutaneous tissue around a central space where the implant would have been located in $50 \%$ of the mice. In two of the 12 animals, small amounts of foreign material, with an associated minimal focal foreign body reaction, was identified in the adipose connective tissue adjacent to the fibrous capsule. These lesions were minor and considered to be of no clinical significance. There was no evidence of generalized or diffuse inflammation, uncontrolled mesenchymal proliferation or tumor formation.

\section{Conclusion}

Insertion of microchips in the groin has no significant effect on the phenotype of mice when assessed using the standard IMPC phenotyping pipeline. While the DEXA results show a significant difference in bone mineral density and bone mineral content between non-chipped and chipped mice, this is to be expected. DEXA relies on transmission of X-rays with high and low energies through a medium to determine the mass per unit (or density) of the medium ${ }^{11}$; RFID microchips in this scenario being a dense hard material behave as bone and skew the results. The microchip therefore affects the results of this test but not the actual bone mineral content or bone mineral density of the mice. However, if microchips are implanted in both test groups and controls this should have no overall effect on the study. The implant does not appear to have any adverse welfare effects on the health of the mice.

The phenotype of an organism is an indicator of how it will function in different environments and under different challenges. Recording the phenotype of a mouse constitutes recording every aspect of clinical, morphological, physiological or cellular change that may arise from any type of experimental manipulation: genetic, surgical or pharmacological ${ }^{12,13}$.

Insertion of microchips in the groin requires the application of general anaesthesia; the post-anaesthetic effects of isoflurane on learning and memory may affect behaviour phenotyping ${ }^{9,14-16}$, although this is unlikely with such brief anaesthesia. The current study does not detect any noticeable effects on the phenotype of the mice. However, the data is limited to a single inbred wild type strain; genetically modified mice with unknown phenotypes may react differently to the required exposure to anaesthesia.

\section{Data availability \\ Underlying data}

Harvard Dataverse: The effects of microchipping C57BL/6N mice on standard phenotyping tests. https://doi.org/10.7910/DVN/ PFLBYU ${ }^{10}$

Data are available under the terms of the Creative Commons Zero "No rights reserved" data waiver (CC0 1.0 Public domain dedication).
1. Loos M, Koopmans B, Aarts E, et al.: Sheltering behavior and locomotor activity in 11 genetically diverse common inbred mouse strains using home-cage monitoring. PLOS One. 2014; 9(9): e108563. PubMed Abstract | Publisher Full Text | Free Full Text

2. Hong W, Kennedy A, Burgos-Artizzu XP, et al:: Automated measurement of mouse social behaviors using depth sensing, video tracking, and machine learning. Proc Natl Acad Sci U S A. 2015; 112(38): E5351-E5360. PubMed Abstract | Publisher Full Text | Free Full Text
3. Bains RS, Wells S, Sillito RR, et al.: Assessing mouse behaviour throughout the light/dark cycle using automated in-cage analysis tools. J Neurosci Methods. 2018; 300: 37-47.

PubMed Abstract | Publisher Full Text | Free Full Text

4. Bains RS, Cater HL, Sillito RR, et al.: Analysis of Individual Mouse Activity in Group Housed Animals of Different Inbred Strains using a Novel Automated Home Cage Analysis System. Front Behav Neurosci. 2016; 10: 106. PubMed Abstract | Publisher Full Text | Free Full Text 
5. Rao GN, Edmondson J: Tissue reaction to an implantable identification device in mice. Toxicol Pathol. 1990; 18(3): 412-416.

PubMed Abstract | Publisher Full Text

6. Le Calvez S, Perron-Lepage MF, Burnett R: Subcutaneous microchip-associated tumours in B6C3F1 mice: A retrospective study to attempt to determine their histogenesis. Exp Toxicol Pathol. 2006; 57(4): 255-265. PubMed Abstract | Publisher Full Text

7. Tillmann $T$, Kamino K, Dasenbrock $C$, et al:: Subcutaneous soft tissue tumours at the site of implanted microchips in mice. Exp Toxicol Pathol. 1997; 49(3-4): 197-200.

PubMed Abstract | Publisher Full Text

8. Lapp S, Bube A, Colbatzky FA, et al: Best Practice Approach for Assessment of Microchip-associated Tumors in Preclinical Safety Studies: Position of the Registry of Industrial Toxicology Animal-data (RITA). Toxicol Pathol. 2018; 46(7): 728-734.

PubMed Abstract | Publisher Full Text

9. Yonezaki K, Uchimoto K, Miyazaki T, et al.: Postanesthetic effects of isoflurane on behavioral phenotypes of adult male C57BL/6J mice. PLOS One. 2015; 10(3): e012211823.

PubMed Abstract | Publisher Full Text | Free Full Text

10. Bains RS: "The effects of microchipping C57BL/6N mice on standard phenotyping tests". Harvard Dataverse, V1, UNF:6:NIHxnYrJ5ZYje5NdBH76Sw== [fileUNF],
2019

http://www.doi.org/10.7910/DVN/PFLBYU

11. Blake GM, Fogelman I: Technical principles of dual energy $\mathbf{x}$-ray absorptiometry. Semin Nucl Med. 1997; 27(3): 210-228.

PubMed Abstract | Publisher Full Text

12. Brown SD, Hancock JM, Gates $H$ : Understanding mammalian genetic systems: the challenge of phenotyping in the mouse. PLoS Genet. 2006; 2(8): e118. PubMed Abstract | Publisher Full Text | Free Full Text

13. Zeiss CJ, Ward JM, Allore HG: Designing phenotyping studies for genetically engineered mice. Vet Pathol. 2012; 49(1): 24-31. PubMed Abstract | Publisher Full Text | Free Full Text

14. Cao L, Li L, Lin D, et al.: Isoflurane induces learning impairment that is mediated by interleukin $1 \beta$ in rodents. PLoS One. 2012; 7(12): e51431. PubMed Abstract | Publisher Full Text | Free Full Text

15. Fidalgo AR, Cibelli M, White JP, et al.: Isoflurane causes neocortical but not hippocampal-dependent memory impairment in mice. Acta Anaesthesiol Scand. 2012; 56(8): 1052-1057.

PubMed Abstract | Publisher Full Text

16. Lee S, Park SH, Zuo Z: Effects of isoflurane on learning and memory functions of wild-type and glutamate transporter type 3 knockout mice. J Pharm

Pharmacol. 2012; 64(2): 302-307.

PubMed Abstract | Publisher Full Text 


\title{
Open Peer Review
}

\section{Current Peer Review Status:}

\section{Version 1}

Reviewer Report 04 February 2020

https://doi.org/10.5256/f1000research.23847.r58613

(C) 2020 Fuchs $\mathbf{H}$ et al. This is an open access peer review report distributed under the terms of the Creative Commons Attribution License, which permits unrestricted use, distribution, and reproduction in any medium, provided the original work is properly cited.

\author{
Helmut Fuchs \\ German Mouse Clinic, Institute of Experimental Genetics, Helmholtz Zentrum München, \\ Neuherberg, Germany

\section{Gregor Miller} \\ German Mouse Clinic, Institute of Experimental Genetics, Helmholtz Zentrum München, \\ Neuherberg, Germany \\ Manuela Östereicher \\ German Mouse Clinic, Institute of Experimental Genetics, Helmholtz Zentrum München, \\ Neuherberg, Germany
}

\section{Annemarie Zimprich (id}

German Mouse Clinic, Institute of Developmental Genetics, Helmholtz Zentrum München, Neuherberg, Germany

The manuscript "The effects of microchipping C57BL/6N mice on standard phenotyping tests" by Bains et al. describes the comparison of phenotyping data from 12 male and 12 female mice that were microchipped in the groin with unchipped animals (10 males and 10 females). The mice were wild-type C57BL/6N and were analyzed in the IMPC phenotyping pipeline. The experiment is highly interesting, as in the mouse phenotyping community there is a lot of effort put in the development of home cage monitoring systems for an undisturbed observation and analysis of mouse behavior in their normal environment. State of the art technologies require microchipping of the mice for a proper identification of individual mice in the system. Thus, it is of importance to investigate the impact of microchips on other tests that are carried out within the IMPC phenotyping pipeline.

The experiments and results are well described in the manuscript, however, there remain some questions about the selected methodology and the possible conclusions: we are missing a clear conclusion in the abstract. In the conclusion part there are three statements: "Insertion of microchips in the groin has no significant effect on the phenotype of mice when assessed using the standard IMPC phenotyping pipeline". "The implant does not appear to have any adverse welfare effects on the health of the mice". The third conclusion is "The current study does not 
detect any noticeable effects on the phenotype of the mice". How far is it via the selected approach possible to obtain information about effects of microchipping on other phenotyping tests - or in other words: how probable is it that there are really no differences if under the given conditions and analysis no differences were detected? For example, the neurological, behavioral and sensory analysis data: The authors decided for an alpha value of $1 \%$ and in addition did a correction for multiple testing. Thus, the observed differences need to be very high to reach statistical significance.

Additional comments:

Statistical analysis: On which parameters and which tests was the sample size and power calculation based? More detailed information on the power calculation would be desirable. Is there a special reason why the authors select an alpha value of $1 \%$ ? Also, a more detailed description on the method for multiple testing correction would be helpful.

Maybe the results part should reflect the order of the tests in the phenotyping pipeline. For a couple of tests from the mentioned phenotyping pipeline there are no results mentioned. For example, in the open-field and SHIRPA analysis the authors report only data from locomotion related parameters. What about the other areas that are covered by these tests (e.g. anxiety and exploratory behaviors)?

Body weight: According to the provided graphs, at the age of chip implantation (7 weeks) the body weight data flips. In females, non-chipped mice are below, and afterwards are heavier than chipped mice. In males, non-chipped mice are below the chipped mice and then are heavier. Is there any idea about the reason? What is the weight of the RFID? Can the weight of the chip itself make a difference in body weight?

It might be interesting to know whether the authors also considered other types of RFID's for the experiment, and why they selected the used one for the study?

DEXA analysis: Is it possible to cut out the area that is covered by the chip from the DEXA image for the analysis? A DEXA-Image of a mouse with chip compared with an unchipped mouse might be helpful.

Is there more detailed information available about the material contained inside the chip? If it contains some metal, it might cause scattered radiation that could be harmful for the mouse, and if the DEXA-system is not completely shielded (as it might be the case for older DEXA-systems), also for the experimenter.

Is the work clearly and accurately presented and does it cite the current literature? Yes

Is the study design appropriate and is the work technically sound? Partly

Are sufficient details of methods and analysis provided to allow replication by others? Partly

If applicable, is the statistical analysis and its interpretation appropriate? Partly 
Are all the source data underlying the results available to ensure full reproducibility? Yes

Are the conclusions drawn adequately supported by the results? Partly

Competing Interests: The authors are members of the International Mouse Phenotyping Consortium (IMPC), as well as we are. The research presented in the manuscript is not directly part of the IMPC work, but will serve to bring the project further. We do not believe this has affected our ability to write an objective and unbiased review of the article.

Reviewer Expertise: Mouse phenotyping

We confirm that we have read this submission and believe that we have an appropriate level of expertise to confirm that it is of an acceptable scientific standard, however we have significant reservations, as outlined above.

Author Response 13 Mar 2020

Rasneer Sonia Bains, Mary Lyon Centre, Harwell Campus, UK

Response: We thank the reviewers for their comments and suggestions on our manuscript. We have tried to address the concerns raised in the responses below and would be amending the manuscript to reflect these changes.

The manuscript "The effects of microchipping C57BL/6N mice on standard phenotyping tests" by Bains et al. describes the comparison of phenotyping data from 12 male and 12 female mice that were microchipped in the groin with unchipped animals (10 males and 10 females). The mice were wild-type C57BL/6N and were analyzed in the IMPC phenotyping pipeline. The experiment is highly interesting, as in the mouse phenotyping community there is a lot of effort put in the development of home cage monitoring systems for an undisturbed observation and analysis of mouse behavior in their normal environment. State of the art technologies require microchipping of the mice for a proper identification of individual mice in the system. Thus, it is of importance to investigate the impact of microchips on other tests that are carried out within the IMPC phenotyping pipeline.

The experiments and results are well described in the manuscript, however, there remain some questions about the selected methodology and the possible conclusions: we are missing a clear conclusion in the abstract.

Response: The authors will add the following text in the abstract: "No significant impact of insertion of microchip was observed in any of the phenotyping tests apart from bone mineral density measurement at DEXA due to the nature of the microchip. We therefore recommend that the microchip be inserted during the DEXA procedure, after the measurement is taken but before the mouse has recovered from the anaesthetic. This would avoid multiple anaesthetic exposures and prevent the potential variability in DEXA analysis output."

In the conclusion part there are three statements: "Insertion of microchips in the groin has 
no significant effect on the phenotype of mice when assessed using the standard IMPC phenotyping pipeline". "The implant does not appear to have any adverse welfare effects on the health of the mice". The third conclusion is "The current study does not detect any noticeable effects on the phenotype of the mice". How far is it via the selected approach possible to obtain information about effects of microchipping on other phenotyping tests or in other words: how probable is it that there are really no differences if under the given conditions and analysis no differences were detected? For example, the neurological, behavioral and sensory analysis data: The authors decided for an alpha value of $1 \%$ and in addition did a correction for multiple testing. Thus, the observed differences need to be very high to reach statistical significance.

Response: We agree with the concerns raised by the reviewers about the stringent criteria, however, the aim of the current study was to establish the effect of a single procedure on a whole pipeline. We therefore decided to choose the middle ground between using an alpha of $5 \%$ and $0.01 \%$, without compromising the integrity of the study.

Additional comments:

Statistical analysis: On which parameters and which tests was the sample size and power calculation based? More detailed information on the power calculation would be desirable. Is there a special reason why the authors select an alpha value of $1 \%$ ? Also, a more detailed description on the method for multiple testing correction would be helpful.

Response: As discussed in Karp et al., 2015, the calculation of false discovery rate is an ongoing discussion within the consortia. While the current work draws from the ARRIVE guidelines published in Karp et al., 2015, it was felt that as the current study was only carried out in one centre and over a short period of time using an alpha of 0.0001 would perhaps be too stringent and lead to a type 2 error. We therefore chose an alpha value of $1 \%$. Based on an alpha of 0.01 and $\mathrm{R}=11$ tests the alpha was calculated to be 0.001 , and not 0.005 as initially reported in the manuscript. We thank the reviewers for picking up this typo, we would like to add here that this does not alter the results. We will correct the text to reflect this.

Maybe the results part should reflect the order of the tests in the phenotyping pipeline. For a couple of tests from the mentioned phenotyping pipeline there are no results mentioned. For example, in the open-field and SHIRPA analysis the authors report only data from locomotion related parameters. What about the other areas that are covered by these tests (e.g. anxiety and exploratory behaviors)?

Response: It was not possible, given the character limit to include every parameter in the main body of the text, however all data points have been included in the data repository. Locomotor activity was chosen as the representative parameter as this was the most likely aspect of the mouse behaviour to be affected by insertion of a microchip to the groin. The other biological system most likely to be effected was the metabolism and as such was included in the main body of the text.

Body weight: According to the provided graphs, at the age of chip implantation (7 weeks) the body weight data flips. In females, non-chipped mice are below, and afterwards are heavier than chipped mice. In males, non-chipped mice are below the chipped mice and then are heavier. Is there any idea about the reason? What is the 
weight of the RFID? Can the weight of the chip itself make a difference in body weight?It might be interesting to know whether the authors also considered other types of RFID's for the experiment, and why they selected the used one for the study? Response: The weights when expressed as individual data points show a near complete overlap and there is no difference in the two groups in either sex. The slight changes in trend can be attributed to individual animals that have a low in case of females and high starting weight in case of males, which were then randomly assigned to one of the two groups and have therefore continued to influence the trend through the study period. The RfiDs used in the current study on average weigh approximate $100 \mathrm{mg}$, it is not currently possible to use a wide variety of microchips from different manufacturers as the baseplate readers are tuned to particular chip types and it is not simply a case of swapping one chip for another without tuning and validating the new chips. As the chips used in the current study were used to develop and validate the system we did not deem it necessary to change the chips for the current study.

DEXA analysis: Is it possible to cut out the area that is covered by the chip from the DEXA image for the analysis? A DEXA-Image of a mouse with chip compared with an unchipped mouse might be helpful.Is there more detailed information available about the material contained inside the chip? If it contains some metal, it might cause scattered radiation that could be harmful for the mouse, and if the DEXA-system is not completely shielded (as it might be the case for older DEXA-systems), also for the experimenter.

Response: It is possible to analyse the data as suggested, and we will include this suggestion in our conclusion. In addition, we will also include the images as suggested in the supplementary data with the paper.

While the equipment in our laboratories is well shielded and the harm from scattered radiation is very small. The legislation in the U.K. has approved the microchips for use in pets. We agree with the concerns raised and therefore recommend that the mice be chipped after DEXA, before the mice recover from anaesthetic. We will include this in our manuscript.

Competing Interests: No competing interests were disclosed.

Reviewer Report 23 January 2020

https://doi.org/10.5256/f1000research.23847.r58610

(C) 2020 Williams J. This is an open access peer review report distributed under the terms of the Creative Commons Attribution License, which permits unrestricted use, distribution, and reproduction in any medium, provided the original work is properly cited.

\section{Jonathan M. Williams}

Pathobiology and Population Sciences, Royal Veterinary College, North Mymms, UK

In this brief report manuscript, entitled "The effects of microchipping C57BL/6N mice on standard 
phenotyping tests", Bains et al. describe the development of a home cage analysis system which tracks activity mouse activity through a RFID microchip placed in the groin in 12 male and 12 female $c 57 \mathrm{bl} / 6$ mice at 7 weeks of age.

This is of particular value as RFID microchips can measure a large number of phenotypic responses with frequent measurements over large time periods without handling or stress inducement in the animal.

The results demonstrate that importantly, the procedure of chip implantation does not significantly alter across a large number of measurable phenotypic responses - only causing significant changes in measurements of bone density.

Overall this is a well written report.

I have only minor criticisms relating to the methods:

In the husbandry section, it is stated that "While the method of randomisation was simple..."- it is not described. What was the method of randomisation?

Are animals sedated or under general anaesthesia for chip insertion? Under the microchipping section, it states "This procedure was performed on sedated mice" but elsewhere in the manuscript general anaesthesia is frequently referenced.

It also states "we have found that a combination of these anaesthetics is the most refined method of delivering these scientific data, as this minimizes any acute discomfort the animal may feel after recovering from the general anaesthetic."

There are no details of the manufacturer/source of the RFID chip or software used which would present problems in attempting replication of this experiment.

Is the work clearly and accurately presented and does it cite the current literature? Yes

Is the study design appropriate and is the work technically sound?

Yes

Are sufficient details of methods and analysis provided to allow replication by others? Partly

If applicable, is the statistical analysis and its interpretation appropriate?

I cannot comment. A qualified statistician is required.

Are all the source data underlying the results available to ensure full reproducibility? Yes

Are the conclusions drawn adequately supported by the results? Yes 
Competing Interests: No competing interests were disclosed.

Reviewer Expertise: Veterinary anatomic pathology and intestinal disease

I confirm that I have read this submission and believe that I have an appropriate level of expertise to confirm that it is of an acceptable scientific standard.

Author Response 03 Feb 2020

Rasneer Sonia Bains, Mary Lyon Centre, Harwell Campus, UK

We would like to thank the reviewer for taking the time to review our manuscript and for sharing their comments. Please find below our response to the same.

1. In the husbandry section, it is stated that "While the method of randomisation was simple..."- it is not described. What was the method of randomisation?

Response: The method of randomisation was 'simple randomisation', described in the arrive guidelines as, "All animals/samples are simultaneously randomised to the treatment groups without considering any other variable. This strategy is rarely appropriate as it cannot ensure that comparison groups are balanced for factors or covariates that might influence the result of an experiment."

We will clarify that in the text by rewording the statement to "The method of randomisation used was Simple Randomisation..."

2. Are animals sedated or under general anaesthesia for chip insertion? Under the microchipping section, it states "This procedure was performed on sedated mice" but elsewhere in the manuscript general anaesthesia is frequently referenced.

It also states "we have found that a combination of these anaesthetics is the most refined method of delivering these scientific data, as this minimizes any acute discomfort the animal may feel after recovering from the general anaesthetic."

Response: The procedure was performed on anaesthetised mice, we will change the text in this section to reflect that.

3. There are no details of the manufacturer/source of the RFID chip or software used which would present problems in attempting replication of this experiment.

Response: The details of the RFID microchips are supplied in the Microchipping section of the manuscript, "These microchips were contained in standard ISO biocompatible glass capsules (11.5×2mm, PeddyMark Ltd. UK)."

The software used to read these RFID data is described in reference 4 - Bains et al., 2016, where the details of the Home Cage Analysis system (Actual Analytics, Edinburgh) have been published. As the RFID data were not part of the current manuscript, the details of the software were not considered essential to the replicate this study. 
Competing Interests: No competing interests were disclosed.

The benefits of publishing with F1000Research:

- Your article is published within days, with no editorial bias

- You can publish traditional articles, null/negative results, case reports, data notes and more

- The peer review process is transparent and collaborative

- Your article is indexed in PubMed after passing peer review

- Dedicated customer support at every stage

For pre-submission enquiries, contact research@f1000.com 